\title{
Keratocan Expression Is Increased in the Stroma of Keratoconus Corneas
}

\author{
Kelly Wentz-Hunter, E. Lillian Cheng, Jun Ueda, Joel Sugar, and Beatrice Y.J.T. Yue \\ Department of Ophthalmology and Visual Sciences, University of Illinois at Chicago, College of Medicine, \\ Chicago, Illinois, USA \\ Accepted April 5, 2001
}

\begin{abstract}
Background: Keratoconus is a noninflammatory disease characterized by thinning and scarring of the central portion of the cornea. The etiology is unclear. In this study, we sought to identify mRNAs that are differentially expressed in the stroma of keratoconus corneas in comparison to those of corneas from normal individuals and patients with other corneal diseases.

Materials and Methods: Total RNA was isolated from the stromal layer of normal human, keratoconus, and pseudophakic bullous keratopathy corneas. cDNA was synthesized and PCR-select subtractive hybridization experiments were performed. The differentially expressed genes noted were verified by dot blot analysis, cloned, and sequenced. Immunohistochemical staining, in situ hybridization, and/or reverse transcription polymerase chain reaction were used to assess expression of the identified genes at protein and/or
\end{abstract}

mRNA levels in normal, keratoconus, and other diseased corneas.

Results: A number of genes were found to be upregulated in keratoconus specimens. These included heat shock protein 90, decorin, fibronectin, ferritin heavy chain, and keratocan. Among them, keratocan mRNA transcript and protein were demonstrated to be expressed at a higher level specifically in the keratoconus stroma.

Conclusions: Keratocan expression in the stoma was increased in keratoconus corneas. This up-regulation appears to be keratoconus specific. Keratocan is one of the three keratan sulfate proteoglycans in the cornea speculated to be important for structure of the stromal matrix and maintenance of corneal transparency. The overexpressed keratocan may conceivably alter the fibrillogenesis in the stroma, leading to structural defects and contributing to the development of keratoconus.

\section{Introduction}

Keratoconus is a noninflammatory disease characterized by thinning and scarring of the central portion of the cornea $(1,2)$. Its exact cause is unclear, although the pathogenesis may involve genetic $(3,4)$ as well as environmental and behavioral factors $(5,6)$. Keratoconus usually is noted during the second decade of life. No specific treatment exists, except to replace the cornea by transplantation when the patient's vision is beyond correction with contact lenses. This disease is one of the leading reasons for corneal transplantation (7).

The stroma, which comprises over $90 \%$ of the thickness of the cornea, is a highly specialized connective tissue. The stromal matrix is made up of tightly packed orthogonal layers of collagen fibrils and abundant keratan and dermatan sulfate proteoglycans. Cells called keratocytes are dispersed in the stromal lamellae. Collagens are believed to be essential for the strength of the cornea, and the interactions between collagen and proteoglycans contribute to the proper collagen spacing and, in turn, to corneal transparency (8-10). In keratoconus,

Address correspondence and reprint requests to: Dr. Beatrice Y.J.T. Yue, Department of Ophthalmology and Visual Sciences, University of Illinois at Chicago, 1855 W. Taylor Street, Chicago, IL 60612. Phone: 312-996-6125; Fax 312-996-7773; e-mail: U24184@uic.edu the stroma is the site where thinning and scarring occurs.

Corneas obtained from keratoconus patients have been shown to contain less total protein than normal ones $(11,12)$. Studies in our laboratory have also revealed biochemical abnormalities in expression levels of both degradative enzymes and protease inhibitors in keratoconus corneas. Specifically, the levels of enzymes including acid esterase, acid phosphatase (13), and cathepsins B and G (14) are increased, whereas those of inhibitors such as $\alpha 1$ proteinase inhibitor and $\alpha 2$-macroglobulin are decreased $(15,16)$. The mRNA levels are also altered and changes paralleled those of the proteins (17). These findings have led to the hypothesis that the degradative process may be one of the mechanisms affected, leading ultimately to stromal thinning manifested in keratoconus (11).

Using a polymerase chain reaction (PCR)-based subtractive hybridization method, we sought in this study to identify mRNAs that are differentially expressed in the stroma of keratoconus corneas in comparison to the counterparts from normal individuals and patients with other corneal diseases such as Fuchs' corneal dystrophy and pseudophakic bullous keratopathy (PBK). Among the genes identified, keratocan was found to be specifically upregulated in the keratoconus stroma. Keratocan is one of three keratan sulfate proteoglycans (KSPG) in 
the human cornea. It has recently been linked to one form of cornea plana, a condition with decreased refraction from a flattened cornea (18). Our data suggest that overexpression of keratocan may be involved in keratoconus.

\section{Materials and Methods}

PCR Select Subtractive Hybridization

Normal human eyes were obtained from the National Disease Research Interchange, Philadelphia, PA, and the Illinois Eye Bank, Chicago, IL. The donors ranged in age from 15-54 years. They did not have any known ocular disease and the corneas were clear. Corneal buttons from keratoconus patients were obtained either at the time of transplantation from the Cornea Service of the University of Illinois at Chicago or within $24 \mathrm{hr}$ of transplantation from Dr. Theodore Perl, Corneal Associates of New Jersey, through arrangement by the National Keratoconus Foundation, Los Angles, CA. Patients ranged in age from 16-67 years at surgery. Corneal buttons from patients (ranging in age from 36-84 years at surgery) with PBK, aphakic bullous keratopathy, Fuchs' corneal dystrophy, and corneal scar from trauma were obtained from the Cornea Service of the University of Illinois at Chicago to serve as controls.

The central region of normal human corneas was obtained using a $7.5-\mathrm{mm}$ trephine. The endothelial and epithelial layers of normal (14- and 33-year-old), keratoconus (16- and 34-year-old), and PBK (81-yearold) corneas were removed and total RNA of the stromal layer was isolated using Trizol reagent (GIBCO/ BRL, Grand Island, NY, USA) as previously described (14). The poly $\mathrm{A}^{+}$RNA was purified using Oligotex mRNA Mini Kit (Qiagen, Valencia, CA, USA) and quantified by absorbance at $260 \mathrm{~nm}$. Approximately $0.1 \mu \mathrm{g}$ of mRNA of normal, keratoconus, or PBK was used to reverse transcribe cDNA by SMART PCR following the manufacturer's protocol (Clontech, Palo Alto, CA, USA).

PCR-select subtractive hybridization (Clontech) was performed. Briefly, each cDNA library was digested with Rsa I to create blunt end fragments. For each experiment, one cDNA sample (e.g., normal control or PBK) was used as the driver and the other (e.g., keratoconus) as the tester. The PCR-select subtractive hybridization allows identification of cDNA up-regulated in the tester population in comparison to the driver. The tester population was divided in half and further processed by ligation to the blunt ends with either adapter 1 or adapter 2, resulting in two populations of tester cDNAs. These adapter 1 and 2 cDNAs were then hybridized individually with a 30-fold excess of driver cDNA. Each mixture was heat denatured and allowed to anneal. As common cDNAs in the tester formed heterohybrids with the driver cDNAs, the differentially expressed or upregulated ones in the tester remained single stranded and became enriched. A second hybridization was subsequently performed by combining the adapter 1 and 2 hybridization reaction mixtures together with additional excess driver to further enrich the differentially expressed cDNA hybrids. Because these hybrids contained adapter 1 at one end and adapter 2 at the opposite end, preferential amplification of this population was achieved by two rounds of suppression PCR through the use of primers specific for the adapters. cDNAs containing the same adapter at both ends were not amplified in the PCR reaction. The PCR products were subsequently ligated into pGEM-T Easy vector (Promega, Madison, WI, USA), transformed into JM109, and selected through blue/ white screening. To identify genes down-regulated in keratoconus stroma, experiments were performed using the keratoconus CDNAs as the driver and normal human or PBK samples as the tester.

\section{Dot Blot Assay}

The subtracted library was differentially screened by dot blot analysis to eliminate false-positive cDNAs. The subtracted library and a reverse subtracted library were used as probes. PCR amplification of the inserts was completed using T7 and SP6 primers (Promega) located on either side of the pGEM-T easy multiple cloning site. The PCR products were spotted onto a nitrocellulose membrane and crosslinked by UV exposure. The cDNAs (100 ng) from the subtractive hybridization were labeled with $\alpha^{32} \mathrm{P}$ dCTP (Amersham, Arlington Heights, IL, USA) by random primed DNA labeling kit (Roche, Nutley, NJ, USA) and were used to hybridize $\left(1 \times 10^{6} \mathrm{cpm}\right)$ overnight at $42^{\circ} \mathrm{C}$ with the blots. The blots were washed and exposed to x-ray films. The clones confirmed to be differentially expressed were sequenced using the DNA Big Dye Sequencing ABI Prism kit (PE Applied Biochemicals, Foster City, CA, USA) and analyzed by the DNA sequencing facility at the University of Chicago. Sequences obtained were compared with those in the GenBank database using BLAST.

\section{Immunostaining}

Paraffin sections of normal, keratoconus, and other diseased corneas were blocked in $10 \%$ heat-inactivated normal goat serum and incubated with polyclonal anti-decorin (1:250, US Biologicals, Swampscott, MA, USA), anti-ferritin heavy chain (1:200, gift from Dr. James Connor [19]), anti-fibronectin (1:100, Cappel, Aurora, OH, USA), or anti-keratocan (1:100) for $1 \mathrm{hr}$ as described previously (20). Corneal sections serving as negative controls received the same dilutions of normal rabbit IgG.

Polyclonal anti-keratocan was raised in rabbits against a synthetic peptide corresponding to the amino acid sequence HLQHLHLDHNK (keratocan amino acids 283-293, GenBank accession number AAC17741, Medline 2002978). The peptide was coupled to keyhole-limpet hemocyanin via a carboxyterminal cysteine residue not present in keratocan. The synthetic peptide was prepared and antibody 
raised by Alpha Diagnostic International (San Antonio, TX, USA). Anti-keratocan was purified by affinity column and the specificity was confirmed by enzyme-linked immunosorbant assay (ELISA). Western blot analysis using anti-keratocan against keratan sulfate proteoglycan extracted from human corneas (a generous gift of Dr. James Fundergurgh, University of Pittsburgh) yielded a 37-kD band not immunoreactive to anti-decorin (data not shown).

After primary antibody incubation, the sections were incubated sequentially with biotinylated goat anti-rabbit IgG (Jackson ImmunoResearch Laboratories, Inc., West Grove, PA, USA), $0.3 \% \mathrm{H}_{2} \mathrm{O}_{2}$ methanol, and avidin-biotin-horseradish peroxidase complex (Vector, Burlingame, CA, USA) each for 30 min. Following color development with 3,3diaminobenzidine tetrahydrochloride (Sigma Chemicals, St. Louis, MO, USA), the sections were dehydrated and mounted in Permount (Fisher Scientific, Itasca, IL, USA). All tissue sections and negative controls were stained simultaneously under identical conditions. Comparisons were made on only sections stained in the same experiment and the immunostaining was repeated at least three times to confirm the results.

\section{In Situ Hybridization}

Corneal specimens were fixed in $4 \%$ formaldehyde for $24 \mathrm{hr}$, processed, and embedded in paraffin. Prior to the experiment, $5-\mu \mathrm{m}$ thick sections were baked at $65^{\circ} \mathrm{C}$ for $1 \mathrm{hr}$, deparaffinized, and rehydrated. The sections were digested with proteinase $\mathrm{K}(10 \mu \mathrm{g} / \mathrm{ml}$, Promega), postfixed with $4 \%$ paraformaldehyde, and treated with $0.25 \%$ acetic anhydride (17). After washing in $2 \times$ sodium chloridesodium citrate buffer, the slides were air dried and hybridized overnight at $42^{\circ} \mathrm{C}$ with digoxigeninlabeled keratocan cDNA probe $(0.5 \mu \mathrm{g} / \mathrm{ml})$ prepared using the Roche PCR DIG labeling kit and primers T7 and SP6. The tissues were further incubated with alkaline phosphatase-conjugated anti-digoxigenin (Roche) and color was developed by reacting with
NBT/BCIP (Sigma). The experiment was repeated at least three times.

\section{Relative Quantitative Reverse Transcriptase PCR}

Total stromal RNA $(0.5 \mu \mathrm{g})$ from corneas of normal individuals (aged 20, 22, 35, 45, and 54 years), and patients with keratoconus $(25,34,36,45$, and 62 years), PBK (68 and 86 years), Fuchs' corneal dystrophy (51 and 80 years), and corneal scar (36 years) was reverse transcribed into cDNA using random hexamer primers and SuperScript II (Roche). Relative reverse transcriptase PCR (RT-PCR) was conducted using the QuantumRNA classical ribosomal 18S primer kit (Ambion, Austin, TX, USA) with keratocan specific primers 5'-GAATTGAAAAAGGAGCCCTAA-3' and 5'-CGGAGGTAGCGAAGATGAG-3' and a 2:8 ratio of $18 \mathrm{~S}$ primers to $18 \mathrm{~S}$ copetimers. PCR was carried out as follows: one cycle $94^{\circ} \mathrm{C}, 2 \mathrm{~min}$ followed by $27 \mathrm{cy}-$ cles, $94^{\circ} \mathrm{C}, 10 \mathrm{sec} ; 62^{\circ} \mathrm{C}, 30 \mathrm{sec} ; 72^{\circ} \mathrm{C}, 2 \mathrm{~min}$ followed by one cycle, $72^{\circ} \mathrm{C}, 7 \mathrm{~min}$. The expected sizes of the keratocan and 18S PCR products were 645- and 488base pairs (bp), respectively. A sample containing only total RNA was used as a negative control. Samples were run on ethidium bromide-stained agarose gels and quantified by densitometry. The level of keratocan expression was normalized to the $18 \mathrm{~S}$ product in each specimen.

\section{Results}

Subtractive hybridization reactions were completed using cDNAs from stromal tissues of normal human, keratoconus, and PBK corneas. A total of four subtractive hybridization reactions in two sets of experiments were done. In each set, keratoconus samples were examined in two reactions against either normal human or PBK stroma. When a clone or a mRNA transcript was identified from both reactions, it was judged more likely to be keratoconus specific and further pursued.

Table 1 summarizes the cDNA samples used and the number of clones that were found differentially expressed. A total of 252 clones were observed to be

Table 1. Summary of subtraction experiments performed on corneal stromal tissue cDNA.

Number of Clones Identified

\begin{tabular}{|c|c|c|c|c|}
\hline \multirow[b]{2}{*}{ Experiments } & \multirow[b]{2}{*}{ Tester } & \multirow[b]{2}{*}{ Donor } & \\
\hline & & & Up-regulated & Down-regulated \\
\hline 1 & $\begin{array}{l}\text { Keratoconus } \\
\text { (16-year-old) }\end{array}$ & $\begin{array}{l}\text { Normal } \\
\text { (14-year-old) }\end{array}$ & 17 & 2 \\
\hline 2 & $\begin{array}{l}\text { Keratoconus } \\
\text { (16-year-old) }\end{array}$ & $\begin{array}{l}\text { PBK } \\
\text { (81-year-old) }\end{array}$ & 21 & 3 \\
\hline 3 & $\begin{array}{l}\text { Keratoconus } \\
\text { (34-year-old) }\end{array}$ & $\begin{array}{l}\text { Normal } \\
\text { (33-year-old) }\end{array}$ & 19 & 1 \\
\hline 4 & $\begin{array}{l}\text { Keratoconus } \\
\text { (34-year-old) }\end{array}$ & $\begin{array}{l}\text { PBK } \\
\text { (81-year-old) }\end{array}$ & 17 & 3 \\
\hline
\end{tabular}


up-regulated when keratoconus samples were used as the tester and 142 to be down-regulated when keratoconus samples were used as the driver. Only 74 up-regulated and 9 down-regulated clones were subsequently confirmed by dot blot analyses (Fig. 1). The down-regulated clones were revealed by sequence analyses to be all mitochondrial genes. Of the upregulated clones, 30 corresponded to novel sequences not found in the database. The remaining clones represented 7 known genes that included heat shock protein 90 (3 clones), decorin (8 clones), fibronectin ( 2 clones), ferritin heavy chain (2 clones), keratocan (16 clones), mitochondrial gene (7 clones), and ALU subfamily (6 clones). Because mitochondrial and ALU genes have been shown to be nonspecific artifacts frequently pulled out in differential display (21), subtractive hybridization (22) and yeast two-hybrid experiments (23), they were not investigated further.

Immunostaining experiments using antibodies for decorin, fibronectin, ferritin heavy chain, and keratocan were carried out. Figure 2 depicts the staining pattern for decorin in normal, keratoconus, and other disease corneas. Positive decorin immunoreactivity was observed in both keratocytes and matrices of normal human corneal stroma (Fig. 2A). The staining was increased especially in the stromal matrix in keratoconus (Figs. 2C and 2E). A similar increase was also observed in corneas of other diseases (Fig. 2F). This pattern was likewise noted for fibronectin and ferritin heavy chain (photographs not shown). The increased expression of these genes thus appeared to be related to disease conditions and was not keratoconus specific.

Positive keratocan staining (Fig. 3) was observed in the stroma of all normal and other diseased corneas. The staining in keratoconus corneas was much stronger throughout the stroma in both keratocytes and matrix lamella (Figs. 3C and 3D) than in normal (Fig. 3A) and disease controls (Figs. 3E and 3F).

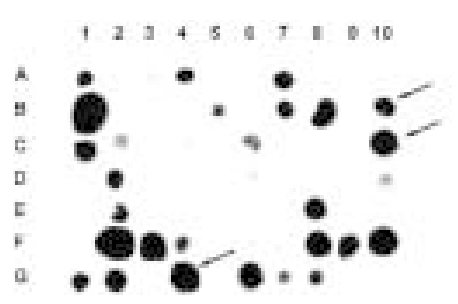

positive

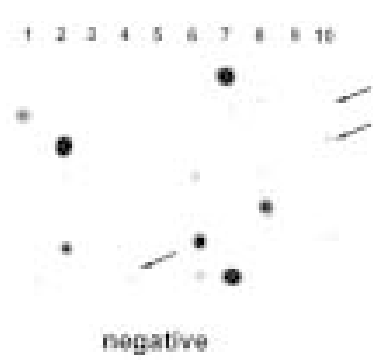

Fig. 1. A representative differential screening dot blot of subtractive hybridization clones. Dot blot analysis was completed on clones isolated from subtractive hybridization experiments. Blots were probed with the subtracted library "positive" and the reverse subtracted library "negative" $\left(10^{6} \mathrm{cpm}\right.$ per $100 \mathrm{ng}$ of subtracted cDNA probe) in ExpressHyb overnight at $65^{\circ} \mathrm{C}$. After washing, the membranes were autoradiographed overnight at $-70^{\circ} \mathrm{C}$. The positions of the clones are marked by numbers $1-10$ and letters $A-G$. Clones identified through sequence analysis to encode portions of the keratocan gene that are up-regulated in the "positive" subtracted library (compared to the "negative") are depicted by the arrows.

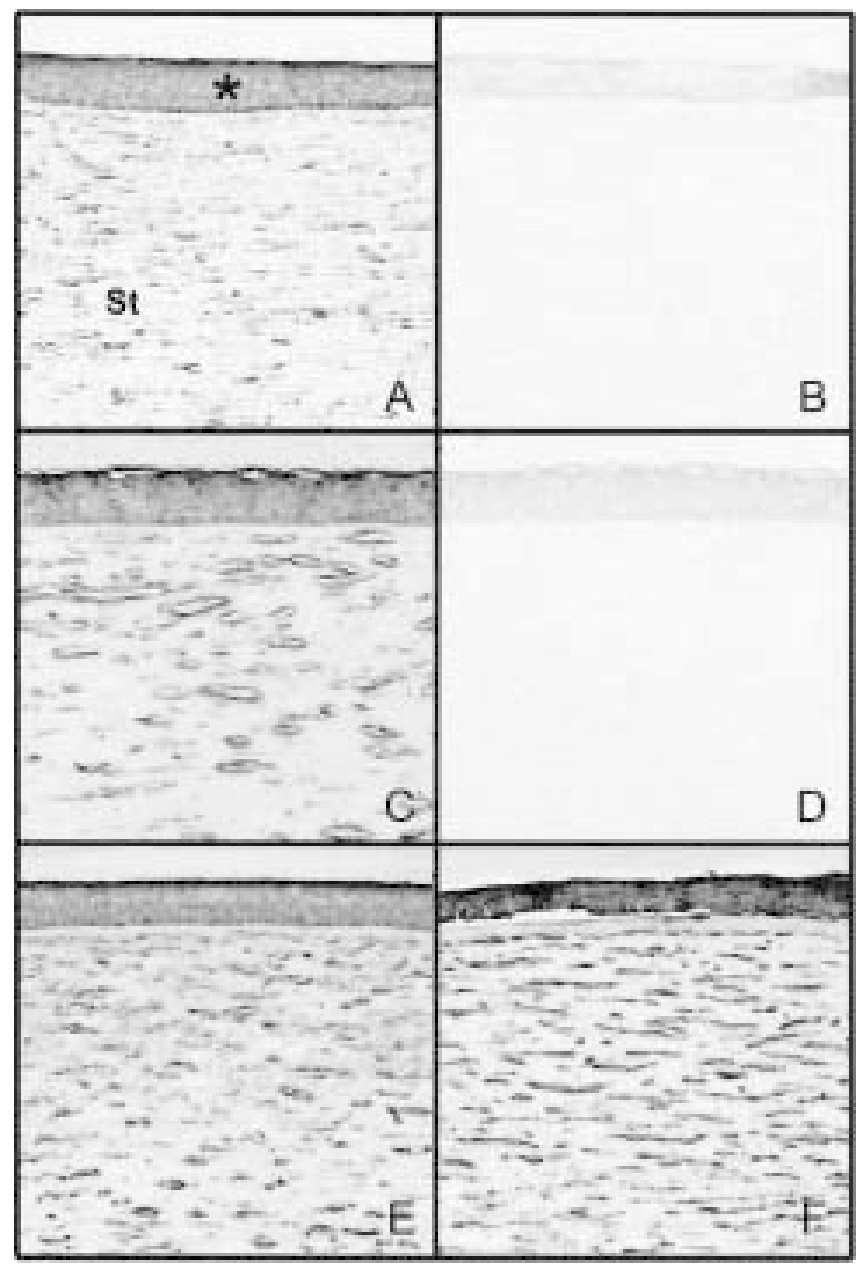

Fig. 2. Immunostaining for decorin. Corneal sections were from a 31-year-old normal human donor (A and B), two patients with keratoconus (C and D, 26-year-old; E, 67-year-old), and a 77-year-old patient with pseudophakic bullous keratopathy (F). Sections were stained with anti-decorin (A, C, E and F) or normal sheep IgG (B and D, negative controls). Positive staining appearing as brown deposits is observed in the corneal stroma (St) as well as the epithelium $\left({ }^{*}\right)$. Note that the staining in the stroma is abnormally increased in diseased corneas (original magnification, $\times 40)$.

Because of the specific increase in immunostaining observed and the fact that keratocan was pulled out multiple times in all subtractive hybridization experiments, investigation was continued with emphasis placed on the keratocan gene. In situ hybridization (Fig. 4) using a keratocan cDNA probe was performed. Blue staining, indicative of positive hybridization products, were observed in keratocytes of all corneas studied. In keratoconus corneas (Figs. 4D, 4E, and 4F), the labeling was visibly enhanced in keratocytes versus normal human (Figs. 4A and 4C) and other disease counterparts (Figs. 4G, 4H, and 4I). A low level of hybridization observed in the epithelial layer of specimens was also noted in negative controls and was judged to be nonspecific. 


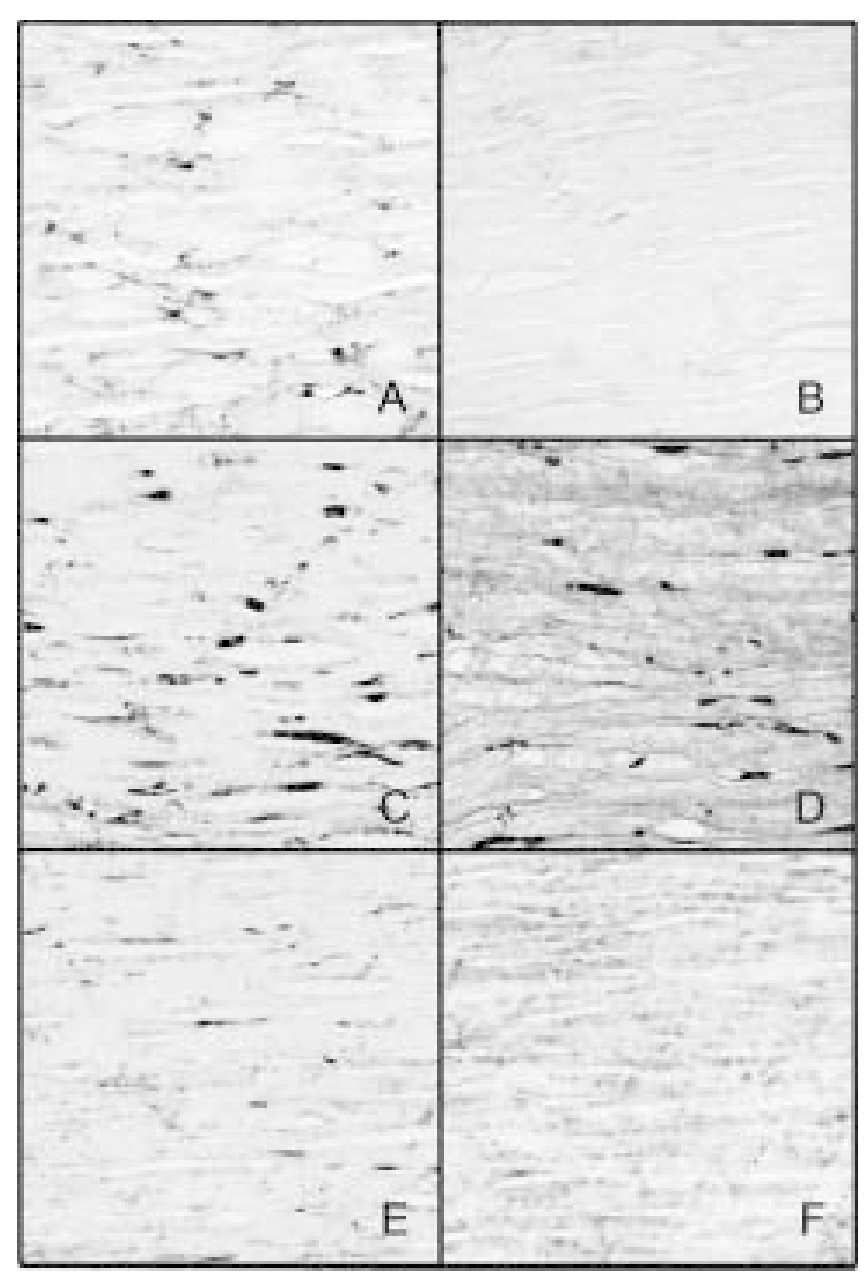

Fig. 3. Immunostaining for keratocan in the corneal stroma. Sections were from a 16-year-old normal individual (A and B), two patients with keratoconus (C, 29-year-old, and D, 67-year-old), an 84-year-old patient with aphakic bullous keratopathy (E), and a 69-year-old patient with corneal scar (F). Sections were immunostained with an antibody against keratocan (A, C, D, E, and F) or normal rabbit IgG (B, negative control). Positive staining appears as brown deposits in both keratocytes and stromal matrices. Note the increased staining in keratoconus specimens (original magnification, $\times 40$ ).

For a more quantitative assessment of the keratocan mRNA level, relative quantitative RT-PCR was completed using stromal RNAs from various corneas. The gel electrophoretogram (Fig. 5) after RT-PCR displayed two products, a 645-bp band for keratocan and a 488-bp band for 18S rRNA. The ratio from both band intensities were obtained via densitometric analyses. Results summarized in Table 2 indicate that the keratocan mRNA level in keratoconus stroma was approximately 1.8- to 2-fold higher than that in both normal human and other disease controls.

\section{Discussion}

PCR-select subtractive hybridization is a recently developed, powerful technique that allows for iden-

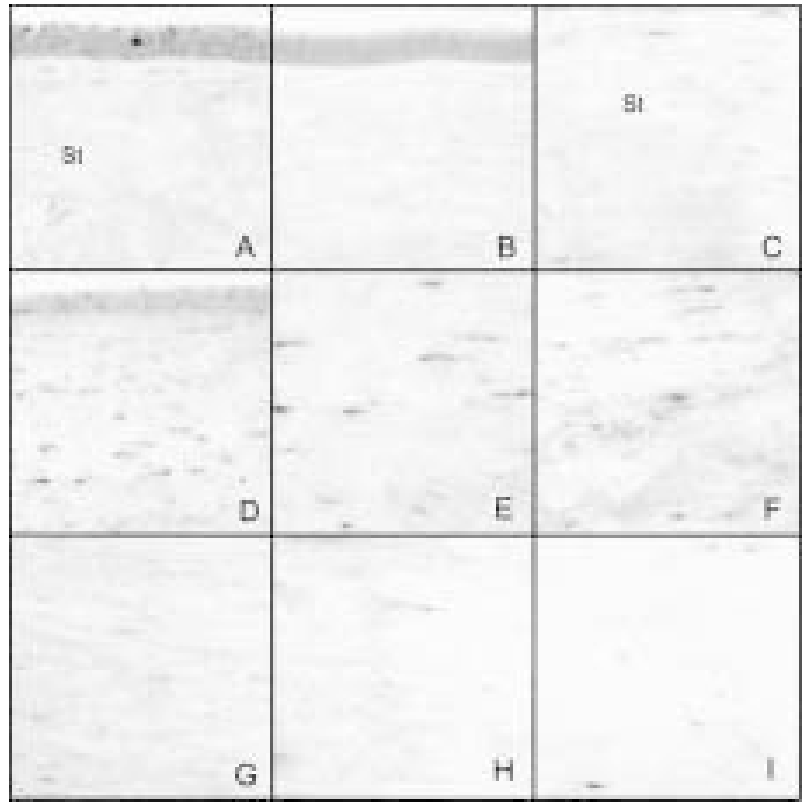

Fig. 4. In situ hybridization using a keratocan cDNA probe. Corneal sections were from a 15-month-old normal donors (A-C), two patients with keratoconus (D and E, 22-year-old; F, 17-year-old), two patients with Fuchs' corneal dystrophy (G, 54-year-old; H, 52-year-old), and a 77-year-old patient with aphakic bullous keratopathy (I). Hybridization without probe served as a negative control in B. Positive hybridization products appear as blue deposits. Note the increase in staining in keratocytes (D-F) of keratoconus specimens compared to those of normal (A and $\mathbf{C}$ ) and other diseased (G-I) corneas. *corneal epithelium; St, stroma. (Original magnification, $\times 10$ for $\mathrm{A}, \mathrm{B}$ and $\mathrm{D} ; \times 40$ for $\mathrm{C}$ and $\mathbf{E}-\mathbf{I})$.

tification of differentially expressed mRNAs. With this method, we detected a number of genes that may be up-regulated in the keratoconus stroma in comparison to normal human and PBK tissues. Apart from novel sequences, seven known genes were identified. Of them, mitochondrial gene and ALU subfamily genes are known to often be nonspecific artifacts (21). Three others-heat shock protein 90, decorin, and fibronectin-have already been evaluated previously in keratoconus (24-26). Work in our laboratory has shown that heat shock protein 90, a member of the stress-response gene family, was not present in normal human, keratoconus, or other diseased corneas (24). The expression of other heat shock protein members was increased in keratoconus, but the increase was not keratoconus specific, and is most likely related to injury repair in disease conditions. Similarly, previous investigation demonstrated an increased decorin expression (25) in the stroma and an increase in fibronectin expression in the epithelium, basement membrane, and the stroma near defect areas in keratoconus (26). Our immunostaining experiments corroborate these findings, and indicate moreover that the enhanced expression of ferritin 


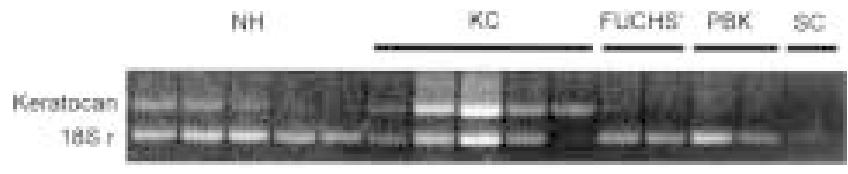

Fig. 5. Relative quantitative RT-PCR of keratocan. Total RNA samples were from 20-, 22-, 35-, 45-, and 54-year-old normal human $(\mathrm{NH})$ donors, 25-, 34-, 36-, 45-, and 62-year-old patients with keratoconus (KC), 51- and 80-year-old patients with Fuchs' corneal dystrophy (FUCHS'), 68- and 86-year-old patients with pseudophakic bullous keratopathy (PBK), and a 36-year-old patient with a corneal scar (SC). The keratocan and 18S rRNA bands are labeled. Total RNA was used as template instead of cDNA as a negative control for relative RT-PCR (lane not shown). No products were detected in the negative control.

heavy chain may also result from wound healing responses seen in all corneal pathologies examined, including keratoconus.

Most significantly, keratocan was found to be up-regulated specifically in the stroma of keratoconus corneas. This result was confirmed by independent methods including immunostaining, in situ hybridization, and relative quantitative RT-PCR.

Table 2. Relative quantitative RT-PCR for keratocan.

\begin{tabular}{lll}
\hline Sample & $\begin{array}{c}\text { Keratocan/18S } \\
\text { Ribosomal RNA } \\
\text { Ratio }\end{array}$ & Mean \pm SD \\
\hline Normal human & 0.84 & \\
$20 y$ & 0.72 & \\
$22 y$ & 0.61 & \\
$35 y$ & 0.67 & \\
$45 y$ & 0.70 & \\
$54 y$ & & \\
Keratoconus & 0.98 & \\
$25 y$ & 1.19 & $1.24 \pm 0.33^{*}$ \\
$34 y$ & 1.10 & \\
$36 y$ & 1.11 & \\
$45 y$ & 1.81 & $0.64 \pm 0.05$ \\
$62 y$ & & $0.52 \pm 0.12$ \\
Fuchs' & 0.67 & \\
$51 y$ & 0.60 & \\
$80 y$ & & \\
PBK & 0.44 & \\
$68 y$ & 0.61 & \\
$86 y$ & & \\
Scar & & \\
$36 y$ & & \\
& & \\
\hline
\end{tabular}

${ }^{*} p<0.008$.

Fuchs', Fuchs' corneal dystophy; PBK, pseudophakic bullous keratopathy; $y$, age of patients in years.
The increase in keratocan expression was not observed in other corneal disease such as PBK or Fuchs' corneal dystrophy. With corneal scars, the levels of keratocan were either comparable to those in normals (Table 2 ) or slightly reduced (Fig. 3F). These data, consistent with previous results in chick corneas that keratocan protein levels remain unaltered during wound healing or in scars (27), argue against the possibility that the increased keratocan expression in keratoconus stroma is related to scarring.

Keratocan is a member of the small leucine rich proteoglycan (SLRP) gene family (28) expressed almost exclusively by corneal keratocytes (29). Along with lumican and mimecan, it makes up the major proteoglycan, KSPG, in the cornea (30-32). The core proteoglycan proteins contain sulfated keratan sulfate side chains in the cornea, whereas in noncorneal tissues, the core proteins lack sulfation (29). KSPGs have long been suggested to be important in corneal transparency and collagen fibrillogenesis $(31,33-35)$. Developmental studies have determined that the emergence of keratan sulfate in the cornea correlates with transparency $(30,36)$, and such a role for KSPG has recently been verified by results from lumican knockout mice that displayed bilateral corneal opacity $(37,38)$. The collagen structure in the posterior stroma was also found altered in the lumicandeficient mice with both increased fibril diameter and abnormal lateral growth (39). Although the specifics have yet to be defined, it is generally believed that keratocan, like lumican, may also take part in regulating both corneal transparency and collagen fibrillogenesis.

Earlier analyses of keratoconus samples have determined that although the amount of keratan sulfate was decreased, the keratan sulfate core protein content remained unaltered (40-42). The KSPG core protein was then considered as only one entity and was measured as such. Therefore, changes in individual KSPGs such as keratocan might have gone unnoticed.

The degradative enzymes, proteinase inhibitors, and transcription factor $\mathrm{Spl}$ previously found to be altered in keratoconus $(13-16,43)$ were not identified in the present study. The subtractive hybridization technique has, in general, a threshold of sensitivity of approximately 5 -fold alterations in transcripts. Although the technique is capable of detecting smaller (as low as 1.5-fold) alterations in expression, the probability of detection diminishes drastically. The messenger RNA level for acid phosphatase has been shown to be only approximately 2 -fold higher and that of $\alpha 1$-proteinase inhibitor is 3-fold lower in keratoconus than the control counterparts (17). Not entirely unexpectedly, neither of these genes was pulled out. Keratocan, on the other hand, was also found to be increased only by approximately 2 -fold. Perhaps by chance, or due to a favorable selection condition, keratocan clones were detected. 
The current study also did not identify the transmembrane phosphotyrosine (LAR) gene recently determined to be up-regulated in keratoconus by Chiplunkar et al. (44) in a differential display investigation. LAR was one of the clones initially isolated in our subtractive hybridization, but was found not to be up-regulated in dot blot screening and was eliminated. In the differential display experiment (44), RNA isolated from corneal stromal cell cultures was used as the starting material. The gene expression in corneal stromal cell cultures (45) may be considerably altered from that in tissue specimens used in our experiments. The differences in the starting material and the techniques may account, at least in part, for the disparity in the results.

To summarize, we used subtractive hybridization and suppression PCR techniques to identify differentially expressed transcripts in keratoconus stromal tissue. These experiments revealed that keratocan, uniquely expressed in the cornea stroma, is up-regulated in keratoconus corneas. Keratocan is one of the three KSPGs in the cornea speculated to be important for structure of the stromal matrix and maintenance of corneal transparency. The overexpressed keratocan may conceivably alter the fibrillogenesis in the stroma, leading to structural defects and contributing to the development of keratoconus.

Recently, keratocan has been linked to another corneal disease, cornea plana (18). This disease is characterized by a flattened cornea and the loss of refraction. Two mutations were identified in the keratocan gene in patients with a severe, recessive form of cornea plana. One mutation replaces the single asparagine residue with serine in the leucine-rich repeat, a characteristic motif in SLRPs. The other mutation results in a stop codon at amino acid 174 and thus a truncated protein. The investigators hypothesized that both mutations would cause either a loss of function or absence of keratocan and a flattened cornea. It is hence of note that a connection of an increased keratocan expression with the corneal protrusion in keratoconus is suggested by the current study. These findings appear to be consistent with the role of keratocan in collagen structure and perhaps the mechanical architecture of the stroma. A keratocan knockout mouse model may ultimately provide insights in this regard.

\section{Acknowledgments}

The authors thank Dr. Theodore Perl, Corneal Associates of New Jersey, West Orange, NJ, for supplying keratoconus corneas; Dr. James Connor, Penn State University College of Medicine, Hershey, PA, for the generous gift of anti-ferritin heavy chain; Dr. James Funderburgh, University of Pittsburgh, for keratan sulfate proteoglycan extract from human corneas; Chan Boriboun for technical assistance; and Kira Lathrop for expert imaging. This work was supported by grants EY03890 and EY05628, core grant EY01792 from the National Eye Institute, Bethesda, MD, and by an award from Research to Prevent Blindness, Inc., New York, to B.Y.J.T.Y.

\section{References}

1. Krachmer JH, Feder RS, Belin MW. (1984) Keratoconus and related non-inflammatory corneal disorders. Surv. Ophthalmol. 28: 293-322.

2. Rabinowitz YS. (1998) Keratoconus. Surv. Ophthalmol. 42: 297319.

3. Rabinowitz YS, Garbus J, McDonnell PJ. (1990) Computerassisted corneal topography in family members of keratoconus. Arch. Ophthalmol. 108: 365-371.

4. Jacobs DS, Dohlman CH. (1993) Is keratoconus genetic? Int. Ophthalmol. Clin. 33: 249-260.

5. Coyle JT. (1984) Keratoconus and eye rubbing. Am. J. Opthalmol. 97: 527-528.

6. Macsai MS, Varley GA, Krachmer JH. (1990) Development of keratoconus after contact lens wear. Patient characteristics. Arch. Ophthalmol. 108: 534-538.

7. Liu E, Slomovic AR. (1997) Indications for penetrating keratoplasty in Canada, 1986-1995. Cornea 16: 414-419.

8. Komai Y, Ushiki T. (1991) The three-dimensional organization of collagen fibrils in the human cornea and sclera. Invest. Ophthalmol. Vis. Sci. 32: 2244-2258.

9. Axelsson I. (1984) Heterogeneity, polydispersity, and physiologic role of corneal proteoglycans. Acta Ophthalmol. 62: 25-38.

10. Hassell JR, Cintron C, Kublin C, Newsome DA. (1983) Proteoglycan changes during restoration of transparency in corneal scars. Arch. Biochem. Biophys. 222: 362-369.

11. Yue BYJT, Sugar J, Benveniste K. (1984) Heterogeneity in keratoconus: possible biochemical basis. Proc. Soc. Exp. Biol. Med. 175: 336-341.

12. Critchfield JW, Calandra AJ, Nesburn AB, Kenney MC. Keratoconus. I. Biochemical studies of normal and keratoconus corneas. Exp. Eye Res. 46: 953-963.

13. Sawaguchi S, Yue BYJT, Sugar J, Gilboy JE. (1989) Lysosomal enzyme abnormalities in keratoconus. Arch. Ophthalmol. 107: 1507-1510.

14. Zhou L, Sawaguchi S, Twining SS, Sugar J, Feder RS, Yue BYJT. (1998) Expression of degradative enzymes and protease inhibitors in corneas with keratoconus. Invest. Ophthalmol. Vis. Sci. 39: 1117-1124.

15. Sawaguchi S, Twining SS, Yue BYJT, Wilson PM, Sugar J, Chan S-K. (1990) $\alpha$ l-Proteinase inhibitor levels in keratoconus. Exp. Eye Res. 50: 549-554.

16. Sawaguchi S, Twining SS, Yue BYJT, et al. (1994) $\alpha 2$ Macroglobulin levels in normal human and keratoconus corneas. Invest. Ophthalmol. Vis. Sci. 35: 4008-4014.

17. Whitelock RB, Fukuchi T, Zhou L, et al. (1997) Cathepsin G, Acid phophatase, and $\alpha$ l-proteinase inhibitor messenger RNA levels in keratoconus corneas. Invest. Ophthalmol. Vis. Sci. 38: 529-534.

18. Pellegata NS, Diezuez-Lucena JL, Joensuu T, et al. (2000) Mutations in KERA, encoding keratocan, cause cornea plana. Nat. Genet. 25: 91-95.

19. Roskans AJI, Connor JR. (1994) Iron, transferrin, and ferritin in the rat brain during development and aging. J. Neurochem. 63: 709-716.

20. Twining SS, Everse SJ, Wilson PM, Yue BYJT, Chan S-K. (1989) Localization and quantification of $\alpha 1$-proteinase inhibitor in the human cornea. Curr. Eye Res. 8: 389-395.

21. Sompayrac L, Jane S, Burn TC, Tenen DG, Danna KJ. (1995) Overcoming limitations of the mRNA differential display technique. Nucl. Acids Res. 23: 4738-4739.

22. Diatchenko L, Lukyanov S, Lau YF, Siebert PD. (1999) Suppression subtractive hybridization: a versatile method for identifying differentially expressed genes. Meth. Enzymol. 303: 349-380. 
23. Gyuris J, Golemis W, Chertov H, Brent R. (1993) Cidl, a human Gl and S phase protein phosphatase that associates with Cdk2. Cell 75: 791-803.

24. Zhou L, Yue BYJT, Twining SS, Sugar J, Feder RS. (1996) Expression of wound healing and stress-related proteins in keratoconus corneas. Curr. Eye Res. 15: 1124-1131.

25. Funderburgh JL, Hevelone ND, Roth MR, et al. (1998) Decorin and biglycan of normal and pathologic human corneas. Invest. Ophthalmol. Vis. Sci. 39: 1957-1964.

26. Tuori A, Virtanen I, Aine E, Uusitalo H. (1997) The expression of tenascin and fibronectin in keratoconus, scarred and normal human corneas. Graefes Arch. Clin. Exp. Ophthalmol. 235: 222-229.

27. Sundarraj N, Fite D, Belak R, et al. (1998) Proteoglycan distribution during healing of corneal stromal wounds in chick. Exp. Eye Res. 67: 433-442.

28. Iozzo RV. (1998) Matrix proteoglycans: from molecular design to cellular function. Annu. Rev. Biochem. 67: 609-652.

29. Corpuz LM, Funderburgh JL, Funderburgh ML, Bottomley GS, Prakash S, Conrad GW. (1996) Molecular cloning and tissue distribution of keratocan. Bovine corneal keratan sulfate proteoglycan. J. Biol. Chem. 271: 9759-9763.

30. Funderburgh JL, Funderburgh ML, Brown SJ, et al. (1993) Sequence and structural implications of a bovine corneal keratan sulfate proteoglycan core protein. Protein 37B represents bovine lumican and proteins $37 \mathrm{~A}$ and 25 are unique. J. Biol. Chem. 268: 11874-11880.

31. Hassell JR, Kimura JH, Hascall VC. (1986) Proteoglycan core protein families. Annu. Rev. Biochem. 55: 539-567.

32. Funderburgh JL, Funderburgh ML, Mann MM, Conrad GW. (1991) Unique glycosylation of three keratan sulfate proteoglycan isoforms. J. Biol. Chem. 266: 14226-14231.

33. Scott JE (1988) Proteoglycan-fibrillar collagen interactions. Biochem. J. 252: 313-323.

34. Schonherr E, Hausser H, Beavan L, Kresse H. (1995) Decorintype I collagen interaction. J. Biol. Chem. 270: 8877-8883.

35. Rada JA, Cornuet PK, Hassell JR. (1993) Regulation of corneal collagen fibrillogenesis in vitro by corneal keratan sulfate proteoglycan (lumican) and decorin core proteins. Exp. Eye Res. 56: 635-648.

36. Funderburgh JL, Caterson B, Conrad GW. (1986) Keratan sulfate proteoglycan during embronic development of the chicken cornea. Dev. Biol. 116: 267-277.

37. Chakravarti S, Magnuson T, Lass JH, Jepsen KJ, LaMantia C, Carroll H. (1998) Lumican regulates collagen fibril assembly: skin fragility and corneal opacity in the absence of lumican. J. Cell. Biol. 112: 987-996.

38. Saika S, Shiraishi A, Saika S, et al. (2000) Role of lumican in the corneal epithelium during wound healing. J. Biol. Chem. 275: 2607-2612.

39. Chakravarti S, Petroll WM, Hassell JR, et al. (2000) Corneal Opacity in lumican-null mice: defects in collagen fibril structure and packing in the posterior stroma. Invest. Ophthalmol. Vis. Sci. 41: 3365-3373.

40. Funderburgh JL, Panjwani N, Conrad GW, Baum J. (1989) Altered keratan sulfate epitopes in keratoconus. Invest. Ophthalmol. Vis. Sci. 30: 2278-2281.

41. Sawaguchi S, Yue BYJT, Chang I, Sugar J, Robin J. (1991) Proteoglycan molecules in keratoconus corneas. Invest. Ophthalmol. Vis. Sci. 32: 1846-1853.

42. Funderburgh JL, Funderburgh ML, Rodrigues MM, Krachmer JH, Conrad GW. (1990) Altered antigenicity of keratan sulfate proteoglycan in selected corneal diseases. Invest. Ophthalmol. Vis. Sci. 31: 419-425.

43. Whitelock RB, Li Y, Zhou L, Sugar J, Yue BYJT. (1997) Expression of transcription factors in keratoconus, a corneathinning disease. Biochem. Biophys. Res. Commun. 235: 253258.

44. Chiplunkar S, Chamblis K, Chwa M, Rosenberg S, Kenney MC, Brown DJ. (1999) Enhanced expression of a transmembrane phosphotyrosine phosphatase (LAR) in keratoconus cultures and corneas. Exp. Eye Res. 68: 283-293.

45. Beales MP, Funderburgh JL, Jester JV, Hassell JR. (1990) Proteoglycan synthesis by bovine keratocytes and corneal fibroblasts: maintenance of the keratocyte phenotype in culture. Invest. Ophthalmol. Vis. Sci. 40: 1658-1663. 